\title{
Nonlinear Polarizability of Erythrocytes in Non-Uniform Alternating Electric Field
}

\author{
Konstantin V. Generalov ${ }^{1}$, Vladimir M. Generalov ${ }^{1}$, Alexander S. Safatov ${ }^{1}$, \\ Alexander G. Durymanov', Galins A. Buryak', Margarita V. Kruchinina², \\ Mikhail I. Voevoda², Andrey A. Gromov² \\ ${ }^{1}$ Federal Budget Research Institution State Research Center of Virology and Biotechnology Vector, Koltsovo, \\ Novosibirsk Region, Russian Federation \\ ${ }^{2}$ Federal State Budgetary Institution of Internal and Preventive Medicine Siberian Branch under the Russian \\ Academy of Medical Sciences, Novosibirsk, Russian Federation \\ Email: general@vector.nsc.ru
}

Received 26 May 2014; revised 25 June 2014; accepted 24 July 2014

Copyright (C) 2014 by authors and Scientific Research Publishing Inc.

This work is licensed under the Creative Commons Attribution International License (CC BY). http://creativecommons.org/licenses/by/4.0/

c) (i) Open Access

\section{Abstract}

Nonlinear polarizability of erythrocytes in non-uniform alternating electric field (NUAEF) was proved theoretically and experimentally by dielectrophoresis method. The paper presents experimental evidence of the nonlinear polarizability of erythrocytes in the non-uniform alternating electric field. The rotation of erythrocyte around its own axis at more than one revolution per second in the non-uniform alternating electric field in the frequency range $10^{5}<f<4 \times 10^{5} \mathrm{~Hz}$ and the electric field intensity $\mathrm{E} \sim 10^{5} \mathrm{mV}$ is the evidence of its nonlinear polarizability. The theoretical analysis of the density of electric charges capable of overcoming the membrane potential was carried out on the basis of statistical mechanics, the thermal equilibrium in which the particle stays. The nonlinear polarizability of the erythrocyte emerges if the voltage on the membrane exceeds $U_{M}(t) \geq 26.2 \mathrm{mV}$, which was theoretically proved. The alternating electric field from the donor erythrocyte with the amplitude exceeding $26.2 \mathrm{mV}$ forms the constant component of the current $\frac{b U_{m}^{2}(t)}{2}$ in the cytoplasm of the recipient erythrocyte whose energy can be considered as a signal one. The nonlinear equivalent electric circuit of the cell was proposed.

\section{Keywords}

Dielectrophoresis, Polarizability, Rotation, Erythrocyte, Nonlinearity 


\section{Introduction}

The study of polarization and deformation of erythrocytes is an urgent problem in the diagnosis of some diseases. The above characteristics are interrelated in their reaction to practically any pathological process in the organism [1]-[4]. The polarization of a cell in an external electric field is accompanied by the displacement of its electric charges relative to the equilibrium position, the formation of an induced dipole moment and, as a result, the overall deformation of the total cell volume [1] [5]. In turn, the deformability of erythrocytes also depends on their viscoelastic properties i.e. total rigidity and viscosity [6]. The deformation of an erythrocyte is obviously limited by its own finite mass, and the displacement of electric charges relative to the equilibrium position is limited by their electrostatic repulsion in the cell closed volume. Thus, these limitations create conditions for the nonlinear polarization of erythrocytes in an external electric field.

The aim of the work was to study the nonlinear polarizability of erythrocytes in NUAEF with an intensity $10^{5} \mathrm{~V} / \mathrm{m}$ and a frequency range of $(100 \div 1000) \mathrm{kHz}$.

\section{Materials and Methods}

Human erythrocytes obtained from whole blood drawn from the donor's vein were used in the study. To conduct the dielectrophoresis analysis, $2 \mathrm{ml}$ of blood were collected with vacutainers in $3.7 \%$ citrate buffer at a ratio of 9:1. Immediately before the experiment, $10 \mu \mathrm{l}$ of blood were diluted in $0.3 \mathrm{M}$ sucrose solution 30 -fold. Specific resistance of $0.3 \mathrm{M}$ sucrose solution was $\rho=5.80 \times 10^{4} \pm 1.16 \times 10^{4} \mathrm{ohm} \cdot \mathrm{m}, \mathrm{pH} 7.0 \div 7.4$. The cells were suspended in $0.3 \mathrm{M}$ sucrose solution to a concentration of $10^{7} \mathrm{~cm}^{-3}$. Blood collection from donors was performed with the approval of the Biomedical Ethics Committee of the Federal Budget Research Institution Research Institute of Therapy, Siberian Branch of Russian Academy of Medical Sciences (Protocol \# 36 of the meeting of September 18, 2012).

Experiments were performed in a measuring cell where NUAEF was created. Detailed description of the measuring cell and the laboratory device as a whole is presented in [6]. Measurements were carried out in the frequency range $F=\left(10^{5} \div 10^{6}\right) \mathrm{Hz}$. The harmonic voltage $U=8 \pm 0.2$ was applied to the electrodes through the capacitor. As a result, the harmonic voltage on the electrodes was lacking the constant component.

Video monitoring and recording of the speed of erythrocyte rotation around its own axis were carried by the position of a typical natural reference point on its surface. The cell turnover period was measured using an electronic clock built into the computer.

\section{Results and Discussion}

\subsection{Experimental Part}

Experimental observations demonstrated a slow rotation of erythrocytes around their own axes with varying frequency in the frequency range of $10^{5}<f<4 \times 10^{5} \mathrm{~Hz}$ and the electric field intensity $E \sim 10^{5} \mathrm{~V} / \mathrm{m}$. Figure 1 shows the dynamics of rotation of a selected individual cell. Measurements showed that its rotation frequency was $\sim 2.5 \mathrm{~Hz}$.

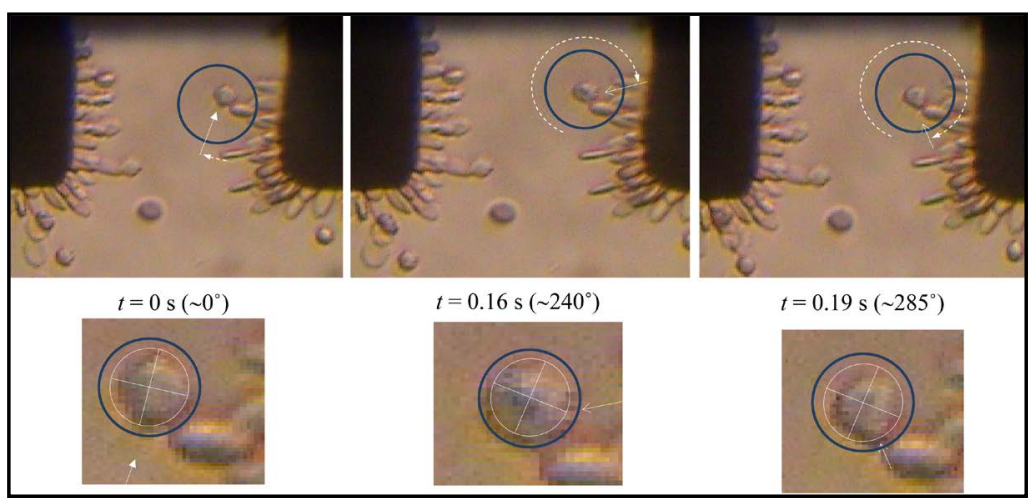

Figure 1. The dynamics of erythrocyte rotation around its own axis in non-uniform alternating electric field. The arrow shows the position of the natural reference point on the cell membrane monitored during the rotation process. 


\subsection{The Oretical Justification}

The external electric field $\boldsymbol{E}_{e x}$ with the frequency $\omega_{e x}$ and the phase $\varphi_{e x}$ applied to the cell induces the redistribution (polarization) of the set $(Q)$ of its free and bound, positive $q^{+}$and negative $q^{-}$charges within the whole cell volume. As a result of polarization, in the cell there emerge uncompensated charges, which create within it its own field with the intensity $\boldsymbol{E}_{c l}$ directed against the external one, $\boldsymbol{E}_{e x}$. The field in the cell volume forms the induced dipole $\boldsymbol{d}_{c l}$, which is the sum of a set of $n$ elementary $\boldsymbol{d}_{c l}=\sum_{i=1}^{n} Q_{i} \cdot 2 \cdot \boldsymbol{r}_{i}$ [5] [9]. In NUAEF, the cell dipole is influenced by the time-averaged force vector, which makes the cell move [10].

$$
\left\langle\boldsymbol{F}_{\text {tot }}\right\rangle=\left\langle 2 \cdot \pi \cdot \varepsilon_{\text {med }} \cdot \varepsilon_{o} \cdot r_{c l}^{3} \cdot\left[\frac{\varepsilon_{c l}-\varepsilon_{\text {med }}}{\varepsilon_{c l}+2 \cdot \varepsilon_{\text {med }}}\right] \cdot \nabla E_{\text {med }}^{2}\right\rangle
$$

where: $\varepsilon_{\text {med }}$ - dielectric permittivity of the medium;

$\varepsilon_{c l}$ - dielectric permittivity of the cell;

$\nabla E_{\text {med }}^{2}$ - the gradient of the square of intensity of the medium electric field;

$\alpha_{c l}=4 \cdot \pi \cdot r_{c l}^{3} \cdot \varepsilon_{m e d} \cdot \frac{\varepsilon_{c l}-\varepsilon_{m e d}}{\varepsilon_{c l}+2 \cdot \varepsilon_{m e d}}$-polarizability coefficient of a spherical cell along a single selected direction, for example, axis $x$.

The vector of the cell electric field intensity $\boldsymbol{E}_{c l}$ with the frequency $\omega_{c l}$ and the phase $\varphi_{c l}$ follows the vector $\boldsymbol{E}_{\text {med }}$

$$
\boldsymbol{E}_{c l}=\boldsymbol{E}_{m}^{c l} \cdot \sin \left(\omega_{c l} \cdot t-\varphi_{c l}\right) .
$$

The superposition of two harmonic oscillations $\omega_{c l}, \omega_{\text {med }}$ results in the emergence of the frequency combinations $\left(\omega_{\text {med }}-\omega_{c l}\right),\left(\omega_{\text {med }}+\omega_{c l}\right)$.

$$
\begin{aligned}
& \boldsymbol{E}_{m}^{\text {med }} \sin \left(\omega_{\text {med }} t-\varphi_{\text {med }}\right)+\boldsymbol{E}_{m}^{c l} \sin \left(\omega_{c l} t-\varphi_{c l}\right) \\
& =2 \boldsymbol{E}_{m}^{\text {med }} \boldsymbol{E}_{m}^{c l} \cos \left(\frac{\left(\omega_{\text {med }}-\varphi_{\text {med }}\right)-\left(\omega_{c l}-\varphi_{c l}\right)}{2} t\right) \sin \left(\frac{\left(\omega_{\text {med }}-\varphi_{\text {med }}\right)+\left(\omega_{c l}-\varphi_{c l}\right)}{2} t\right)
\end{aligned}
$$

The condition $\omega_{c l}=\omega_{\text {med }}$ and $\left|\varphi_{\text {med }}\right|-\left|\varphi_{c l}\right| \leq 90^{\circ}$ is fulfilled for the linear polarization of a cell. If the frequencies $\omega_{c l}=\omega_{\text {med }}$ are equal to each other up to the phase $\varphi_{c l}=\varphi_{\text {med }}$, any rotation around own axis is impossible (2).

The nonlinear polarization of a cell requires the condition $\omega_{c l} \neq \omega_{\text {med }}$ to be fulfilled. If $\left(\omega_{\text {med }}-\omega_{c l}\right) \geq 1 \mathrm{~Hz}$, the cell starts rotating around its own axis, which can be recorded and analyzed by instrumental video monitoring.

The typical value of the erythrocyte transmembrane potential is about $60 \mathrm{mV}$ [11]. This potential creates on the membrane a potential barrier with the electric field intensity $\sim 10^{6} \mathrm{~V} / \mathrm{m}$ whose vector is normal to the cell surface. According to the statistical mechanics, the charge ${ }^{1}$ (ion) should possess the energy required for overcoming the total potential barrier of the cell membrane $W_{m b}(t)$

$$
W_{m b}(t)_{m b}=q^{+} \cdot\left(U_{m}(t) \pm U_{e x}(t)\right)
$$

where:

$q^{+}$- the unit positive charge of the ion $1.6 \times 10^{-19} \quad[\mathrm{~K}]$;

$U_{m}(t)$-the membrane own instantaneous voltage [B];

$U_{\text {med }}(t)$ - the external medium voltage affecting the membrane $U_{\text {med }}(t) \cos \omega_{\text {med }} t \quad[\mathrm{~B}]$.

The density of positive charges capable of overcoming the above barrier is described by Expression [5].

$$
n=n_{o} \cdot e^{\frac{-W_{m}(t) \pm W_{\text {med }}(t)}{k \cdot T}}
$$

where:

$n_{o}$ - the initial density of positive charges on the membrane surface depending the concentration and thermal equilibrium of ions in cell suspension [5];

\footnotetext{
${ }^{1}$ We will consider only positive charges, which move against the forces of electric field formed by the transmembrane potential.
} 
$W_{m}(t)$ - the initial potential energy of the membrane;

$\pm W_{\text {med }}(t)$-the potential energy of the external medium affecting the membrane [J];

$k$-Boltzman constant [J/deg];

$T$ is the absolute temperature $[\mathrm{K}]$.

The exponential function (4) can be determined using the Maclaurin series, which converges at any $\frac{-W_{m}(t) \pm W_{\text {med }}(t)}{k \cdot T}[7]$.

$$
\begin{aligned}
n(x)= & n_{o}\left[1+\frac{\frac{-W_{m}(t) \pm W_{\text {med }}(t)}{k \cdot T}}{1 !}+\frac{\left(\frac{-W_{m}(t) \pm W_{\text {med }}(t)}{k \cdot T}\right)^{2}}{2 !}+\frac{\left(\frac{-W_{m}(t) \pm W_{\text {med }}(t)}{k \cdot T}\right)^{3}}{3 !} \ldots\right. \\
& \left.+\frac{\left(\frac{-W_{m}(t) \pm W_{\text {med }}(t)}{k \cdot T}\right)^{n}}{n !}+\cdots\right]
\end{aligned}
$$

If the summand $\frac{-W_{m}(t) \pm W_{\text {med }}(t)}{k \cdot T} \ll 1$ is much smaller than 1 , the contributions of sum-mands of higher orders (starting from the third, quadratic, one) in (5) can be neglected. As a result, the density of the charges capable of overcoming the potential barrier has a linear character. If the exponent $\frac{-W_{m}(t) \pm W_{\text {med }}(t)}{k \cdot T} \geq 1$ is equal to or more than 1 , the contributions of individual summands of higher orders to nonlinearity become dominant. However, with increasing $n$ value in (5) a general trend is observed: the values of individual summands of the series rapidly decrease.

The interrelations between Expressions $(1,3,4,5)$ allow us to consider the cell polarization process to be nonlinear, too. From the mathematical point of view, nonlinearity in Equation (5) emerges when $U_{m}(t) \geq 26.2 \mathrm{mV}$ and $\frac{-W_{m}(t) \pm W_{\text {med }}(t)}{k \cdot T} \geq 1$. Retaining the summand $W_{\text {med }}(t)$ and proceeding from the classical definition that current $i_{m}(t)$ is determined by the rate of change of the charge $i_{m}(t)=\partial q_{m} / \partial t$, Equation (5) can be rewritten in a form of a sum of currents flowing through the membrane at $W_{m}(t)=0$.

$$
i_{m}(t)=\frac{\partial\left[q^{+} n_{o} \cdot\left[1+\frac{\frac{ \pm W_{\text {med }}(t)}{k \cdot T}}{1 !}+\frac{\left(\frac{ \pm W_{\text {med }}(t)}{k \cdot T}\right)^{2}}{2 !}+\frac{\left(\frac{ \pm W_{\text {med }}(t)}{k \cdot T}\right)^{3}}{3 !} \cdots+\frac{\left(\frac{ \pm W_{\text {med }}(t)}{k \cdot T}\right)^{n}}{n !}+\cdots\right)\right]}{\partial t}
$$

on their serial numbers $n$ are presented in Figure 2, Figure 3. By further simplifying Equation (6), let us reduce it to.

$$
i_{m}(t)=0 \pm a e_{m}(t)+b\left(e_{m}(t)\right)^{2} \pm c\left(e_{m}(t)\right)^{3} \cdots \pm d\left(e_{m}(t)\right)^{n}
$$

where:

$i_{m b}$ - the change in the constant component of the membrane current, equals zero $\frac{\partial\left(q^{+} n_{o}\right)}{\partial t}=0$; 


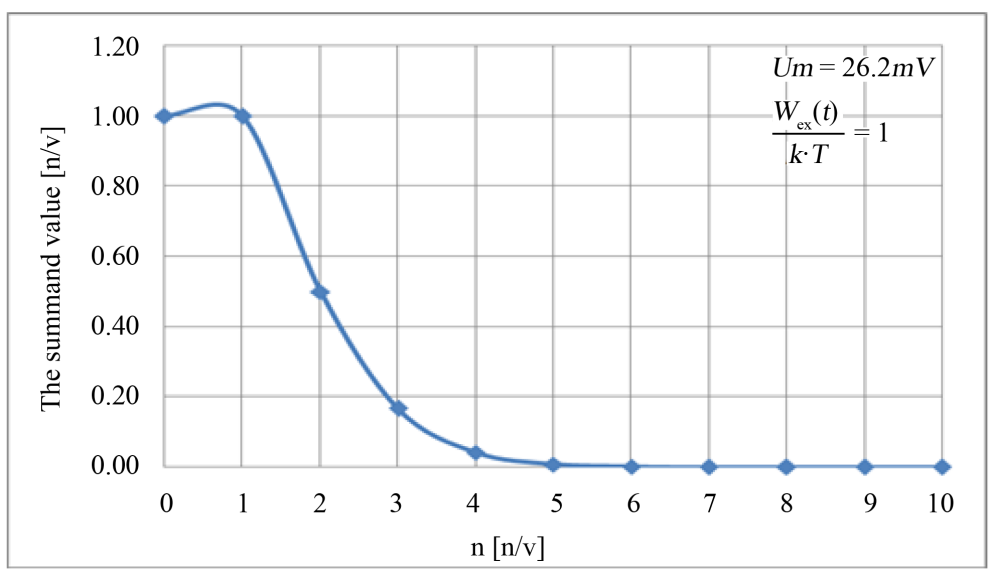

Figure 2. The calculated values $\frac{\left(\frac{ \pm W_{e x}(t)}{k \cdot T}\right)^{n}}{n !}$ of the summands of the Maclaurin series determining the current through the cell membrane depending on $n$ and $U_{m}$, the voltage across the membrane.

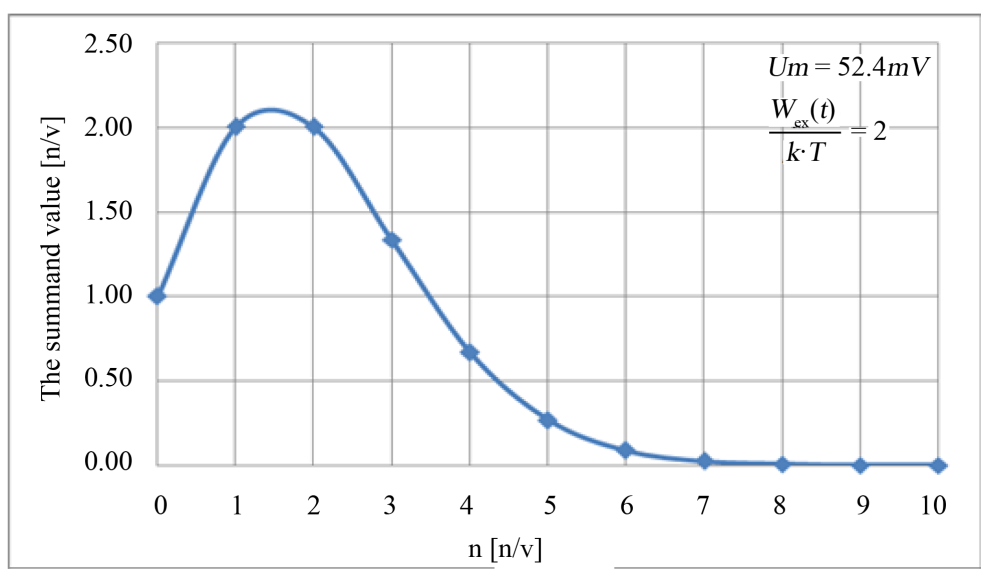

Figure 3. The calculated values $\frac{\left(\frac{ \pm W_{e x}(t)}{k \cdot T}\right)^{n}}{n !}$ of the summands of the Ma-

claurin series determining the current through the cell membrane depending on $n$ and $U_{m}$, the voltage across the membrane.

$e_{m}(t)= \pm U_{\text {med }} \cos \omega_{\text {med }} t$ is the instantaneous voltage of the membrane;

$a, b, c, d$ are the coefficients of proportionality, in particular, $a$ is resistance with the dimension [ohm].

From the consideration of the first three summands of Equation (7) it follows that the alternating current of the membrane contains only the linear and the quadratic components

$$
i_{m}^{\text {med }}(t)=a e_{m}^{\text {med }}(t)+b e_{m}^{2 m e d}(t)=a U_{\text {med }}(t) \cos \omega_{\text {med }} t+b U_{\text {med }}^{2}(t) \cos ^{2} \omega_{\text {med }} t
$$

Taking into account that $\cos ^{2} \omega_{\text {med }} t=\frac{1}{2}+\frac{1}{2} \cos 2 \omega_{\text {med }} t$, let us write

$$
i_{m}^{\text {med }}(t)=a U_{\text {med }}(t) \cos \omega_{\text {med }} t+\frac{b U_{\text {med }}^{2}(t)}{2}+\frac{b U_{\text {med }}^{2}(t)}{2} \cos 2 \omega_{\text {med }} t
$$

The analysis of Expression (8) shows that the total current through the cell membrane is determined by the 
combination of individual harmonic components with frequencies $\omega_{\text {med }}$ and $2 \omega_{\text {med }}$ and the constant component $\frac{b U_{m e d}^{2}(t)}{2}$. This allows us to consider the cell as a nonlinear element, which explains the emergence of the cell currents with the frequency $\omega_{c l} \neq \omega_{\text {med }}$ and that of beat frequencies $\left(\omega_{\text {med }}-\omega_{c l}\right),\left(\omega_{\text {med }}+\omega_{c l}\right)$ determining the cell rotation around its own axis.

The linear model of polarization of the medium and the cell in the external alternating electric field is relatively simple. In each point, the electric field induces in their volume the dipoles with the harmonic frequency $\omega_{e x}$, which coincides with the external one. In the case of the nonlinear polarization, the formation of the induced dipole moment of the cell by electric charges is associated with the fact that their movement is not harmonic any more. In this case, the energy of the cyclic frequency $\omega_{e x}$ of the external field is transferred to the second and higher harmonics, and there also emerge multiple combinations between them [11].

$$
\begin{aligned}
& e(t)=E(t) \cos \omega t \\
& e^{2}(t)=E^{2}(t) \cos ^{2} \omega t=E^{2}(t) \frac{1+\cos 2 \omega t}{2} \\
& e^{2}(t)=E^{2}(t) \sin ^{2} \omega t=E^{2}(t) \frac{1-\cos 2 \omega t}{2} \\
& e^{3}(t)=E^{3}(t) \cos ^{3} \omega t=E^{3}(t) \frac{3 \cos \omega t+\cos 3 \omega t}{4} \\
& e^{3}(t)=E^{3}(t) \sin ^{3} \omega t=E^{3}(t) \frac{3 \sin \omega t-\sin 3 \omega t}{4} .
\end{aligned}
$$

The analysis of the presented known trigonometric expressions also shows that the member of the series with the serial number $n$ in (6) also takes into account the harmonics not of the current flowing through the cell membrane. Thus, in Figure 2, Figure 3, the serial number $n$ along the abscissa agrees with the current harmonics.

Experimental data and the conducted theoretical analysis of the interaction between the cell and alternating electric field suggest that under the study conditions the erythrocyte can be presented as a nonlinear element whose membrane permeability for positive ions is higher in one direction than in the reverse direction. This is consistent with the selective permeability of the membrane, for example, for potassium ions $K^{+}$[12] known from literature. Interestingly, the $p-n$ transition in electronic devices, for example, a diode is characterized by similar different conductivities in different directions [13]. The special case of the nonlinear equivalent electric circuit of the cell for positive charges is shown in Figure 4 where the membrane is presented by a diode with nonlinear resistance and capacity $R_{m} C_{m}$.

The conducted work allowed us to draw the following conclusions.

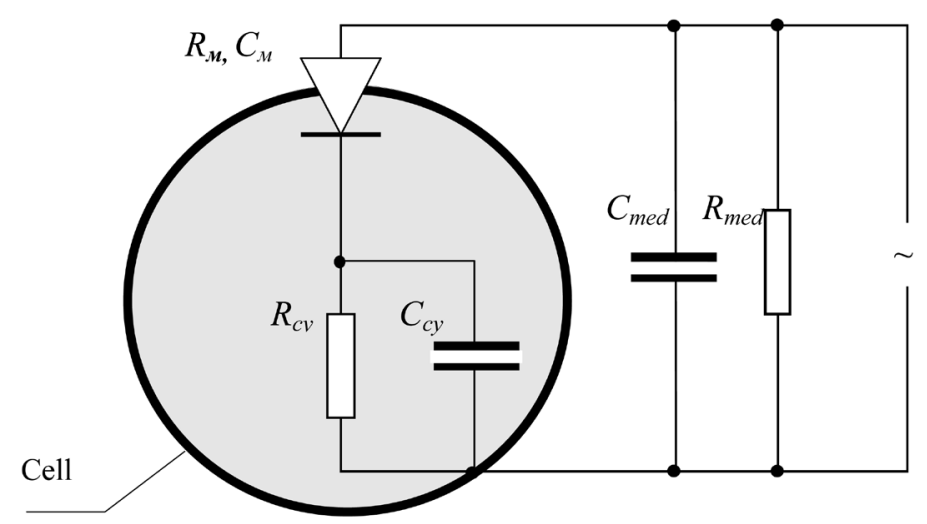

Figure 4. The nonlinear equivalent electric circuit of the cell for positive charges. $C_{m}, C_{c y}$ and $C_{m e d}$ are the capacities of the membrane, cytoplasm and medium (cell suspension); $R_{m}, R_{c y}, R_{\text {med }}$, are the resistances of the membrane, cytoplasm and medium. 


\section{Conclusions}

1) The nonlinear polarizability of human erythrocytes is observed in non-uniform alternating electric field with the intensity $E=10^{5} \mathrm{~V} / \mathrm{m}$ in the frequency range of $(100 \div 1000) \mathrm{kHz}$.

2) The nonlinear polarizability of erythrocytes in non-uniform alternating electric field causes their rotation around their own axes with the frequency exceeding $1 \mathrm{~Hz}$.

3) The external harmonic electric field affecting the cell is created in the cell cytoplasm in the form of a nonlinear uniform ${ }^{2}$ field with a constant component and a broad frequency range due to the electric properties of the cell membrane.

4) The alternating electric field from the donor erythrocyte with the amplitude exceeding $26.2 \mathrm{mV}$ forms the constant component of the current $\frac{b U_{m}^{2}(t)}{2}$ in the cytoplasm of the recipient erythrocyte whose energy can be considered as a signal one.

\section{References}

[1] Zinchuk, V.V. (2001) Erythrocyte Deformability: Physiological Aspects of Progress in Physiological Sciences. Advances in Physiological Sciences, 32, 66-78 (in Russian).

[2] Torkhovskaya, T.I., Artemona, L.G., Khodzhakuliev, B.G., Rudenko, T.S., Polessky, V.A. and Azizova, O.A. (1980) Structural and Functional Changes in Erythrocyte Membranes at Experimental Atherosclerosis. Bulletin of Experimental Biology and Medicine, 89, 675-678 (in Russian). http://dx.doi.org/10.1007/BF00836241

[3] Kruchinina, M.V., Kurilovich, S.A., Parulikova, M.V., Bakirov, T.S., Generalov, V.M., Pak, A.V. and Zvolskiy, I.L. (2005) Electric and Viscoelastic Properties of Erythrocytes of Patients with Diffuse Pathology of the Liver. Proceeding of the Academy of Sciences, 401, 701-704 (in Russian).

[4] Kurilovich, S.A., Kruchinina, M.V., Gromov, A.A., Generalov, V.M., Bakirov, T.S., Rikhter, V.A. and Semenov, D.V. (2010) Justification of the Use of Essential Phospholipids at Chronic Liver Diseases: The Dynamics of Electric and Viscoelastic Parameters of Erythrocytes. Experimental and Clinical Gastroenterology, 11, 46-52 (in Russian).

[5] Feinman, R., Leitos, R., Sands, M. (1977) The Feinman Lectures on Physics. Electricity and Magnetism. Mir., Moscow (in Russian).

[6] Bakirov, T.S., Generalov, V.M. and Toporkov, V.S. (1998) The Measurement of Viscoelastic Properties of a Cell Using the Non-Uniform Alternating Electric Field. Biotechnology, 5, 88-96 (in Russian).

[7] Vorobiev, N.N. (1979) The Theory of Series. Nauka, Moscow, 408 (in Russian).

[8] Generalov, V.M., Bakirov, T.S., Pak, A.V., Zvolskiy, I.L., Zaitsev, B.N., Durymanov, A.G., Kruchinina, M.V., Kurilovich, S.A. and Sergeev, A.N. (2008) The Automated Device for Measurement of Viscoelastic Properties of Erythrocytes. High Technologies, 9, 28-33 (in Russian).

[9] Landau, L.D., Lifshits, E.M. Theoretical Physics (1982) Electrodymanics of Continuous Media. 8, 2th Edition, Nauka, Moscow (in Russian).

[10] Hughes, M.P. (2003) Nanoelectromechanics in Engineering and Biology. CRC PRESS, Boca Raton.

[11] Gelfand, I.M., Lvovsky, S.M., Toom, A.L. Trigonometry. (2002) Moscow, MCCME (in Russia)

[12] Iost, Kh. (1975) Cell Physiology. Mir., Moscow (in Russian).

[13] Lebedev, A.I. (2008) Physics of Semiconductor Devices. Physmathlit, Moscow (in Russian). 
Scientific Research Publishing (SCIRP) is one of the largest Open Access journal publishers. It is currently publishing more than 200 open access, online, peer-reviewed journals covering a wide range of academic disciplines. SCIRP serves the worldwide academic communities and contributes to the progress and application of science with its publication.

Other selected journals from SCIRP are listed as below. Submit your manuscript to us via either submit@scirp.org or Online Submission Portal.
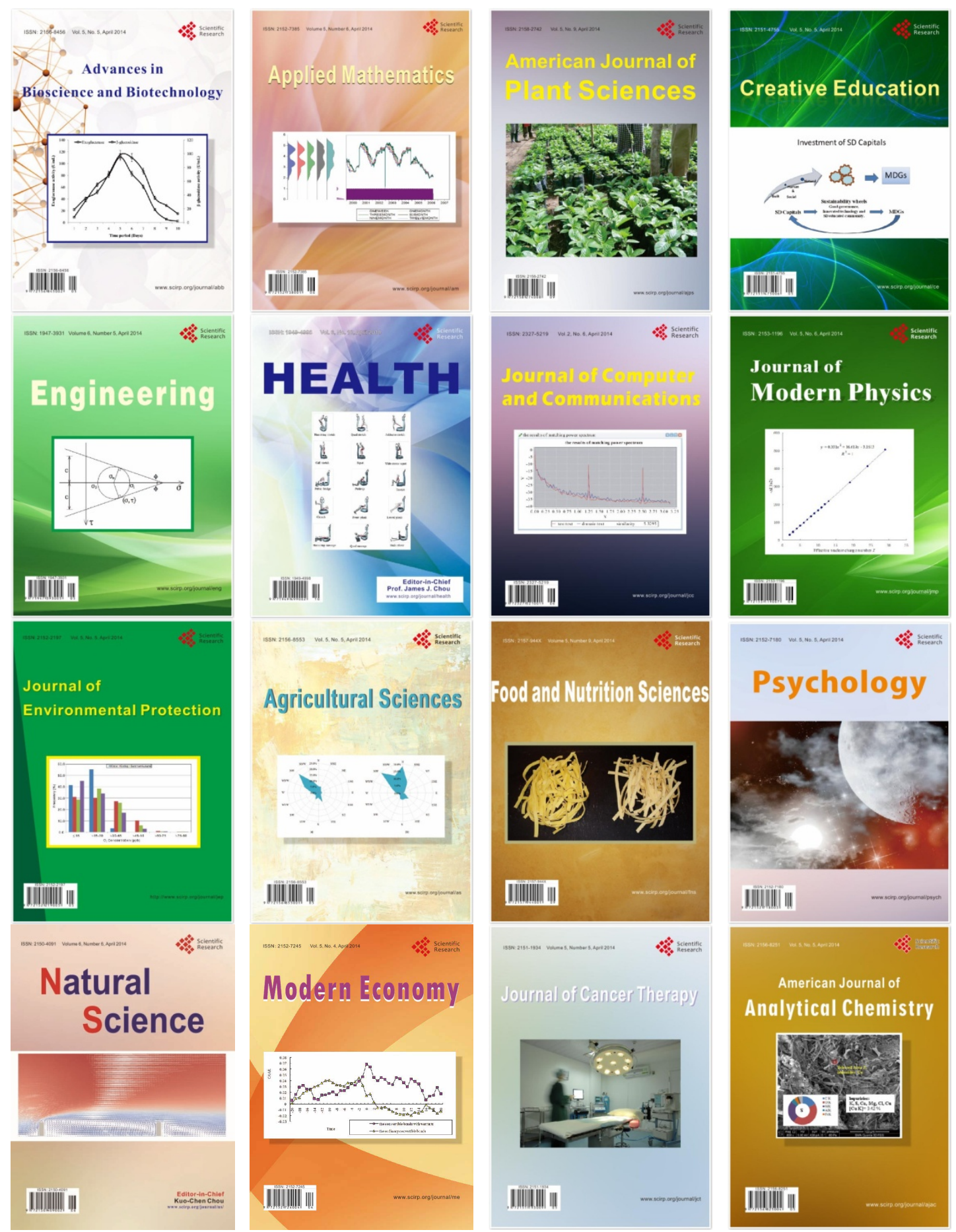